\title{
Vortex Flow Developed in the Vicinity of a Wall of an Elastic Heaving Airfoils and its Wake Structure*
}

\author{
Tomoki KURINAMI**, Masaki FUCHIWAKI*** and Kazuhiro TANAKA*** \\ ${ }^{* *}$ Graduate School of Computer Science and System Engineering, Kyushu Institute of Technology, \\ E-mail: kurinami@vortex.mse.kyutech.ac.jp \\ *** Department of Mechanical Information Science and Technology, Kyushu Institute of Technology, \\ 680-4 Kawazu, lizuka-city, Fukuoka, 820-8502, Japan
}

\begin{abstract}
The authors have previously extensively investigated the flow field around an unsteady airfoil both experimentally and a numerically. In particular, we have performed a number of studies on wake structures and have reported the characteristics of dynamic thrust of an unsteady airfoil. However, the behaviors of vortices that form wake structures and processes from generation to development have not yet been clarified. The authors measured the flow field in the vicinity of a wall including the boundary layers by particle image velocimetry measurements and clarified the growth and development of vortices generated in the vicinity of the wall and the process of wake structure formation quantitatively. We clarified that the vortex flow in the vicinity of the wall and the process of wake structure formation are different between the rigid NACA0010 and the elastic NACA0010, whereas the wake structures for which flow fields are averaged for one heaving cycle become almost the same in the wake of the rigid NACA0010 and the elastic NACA0010.
\end{abstract}

Key words: Vortex, Elastic Airfoil, Vicinity of a Wall, Wake Structure

\section{Introduction}

The authors have previously investigated the flow field around an unsteady airfoil, which is one of the most common unsteady flows, both experimentally and numerically. In particular, since Katzmayr ${ }^{(1)}$ revealed through wind tunnel experiments that a flapping wing generates thrust and lift by creating an effective angle of attack (Knoller-Betz effect), a number of studies on wake structures and the characteristics of dynamic thrust of an unsteady airfoil have been reported. Jones et al. ${ }^{(2)}$ and Lai et al. ${ }^{(3)}$ clarified the vortex flow structures and the generation of dynamic thrust of a plunging airfoil by laser doppler velocimetry (LDV) measurements, as well as numerically using a panel method. Ramamurti et al. ${ }^{(4)}$ calculated the efficiency of a combination airfoil with combined pitching and heaving motions by a finite element method and clarified that the efficiency of the combination airfoil reaches the maximum value when the phase difference of pitching and heaving motions is $90 \mathrm{deg}$.

In addition, we clarified the wake structures and the characteristics of dynamic thrust of a combination airfoil ${ }^{(5)}{ }^{(6)}$. In particular, by combing pitching and heaving motions, we revealed that even if the Strouhal number is low, a combination airfoil forms a thrust producing vortex street clearly in the wake. In addition, the thrust efficiency of the combination airfoil becomes $40 \%$ when the phase difference $\phi$ is $90 \mathrm{deg}$. Most of the relationships between wake structures and the characteristics of dynamic thrust were

${ }^{*}$ Received 3 Jan., 2011 (No. 11-0002) [DOI: 10.1299/jfst.6.562]

Copyright $\mathbb{C} 2011$ by JSME 
clarified by these studies. Therefore, it is important to determine the growth processes of vortex forming wake structures in a wake. However, the behaviors of vortices that form wake structures and the processes from generation to development have not yet been clarified.

Animals are known to fly and swim in a narrow range of Strouhal numbers $(0.2<S t<0.4)^{(7)}$. A flapping robot that mimics the flight mechanism of insects has been recently developed, and an elastic airfoil that enables flying and swimming with low energy has been reported. The flow field around an elastic body is treated as a fluid structure interaction (FSI) problem, and this FSI problem has primarily been investigated numerically, although a few experimental studies have been reported. Heathcote et al. ${ }^{(8)(9)}$ investigated a heaving tadpole-shaped airfoil that deforms elastically and clarified the effects of chordwise flexibility on dynamic thrusts by particle image velocimetry (PIV) measurements and dynamic thrust measurements. Young ${ }^{(10)}$ showed experimental and numerically that the elastic deformation of the marine propeller's blade reduces vibrations and noises without reducing propeller efficiency. However, these studies did not report the details of elastic deformation effects on the flow field or the differences between the flow field of an elastic airfoil and a rigid airfoil. Therefore, we need to clarify the relationship among motion, deformation, and vortex development in order to apply elastic deformation in industry.

The objective of the present paper is to investigate the growth of vorticity of vortices generated in the vicinity of a wall, the wake structures of these vortices, and the behaviors of these vortices generated by applying heaving motions to the elastic NACA0010.In addition, we clarify quantitatively the vortex flows developed in the vicinity of the wall. We measure the flow field in the vicinity of a wall including boundary layers by PIV measurements and clarify quantitatively the growth and development of vortices generated in the vicinity of the wall as well as the process of wake structure formation. We also attempt to determine the effects of elastic deformation on such a growth process of vortices and the behaviors of these vortices through comparing the results with the results obtained for the rigid NACA0010.

\section{Nomenclature}

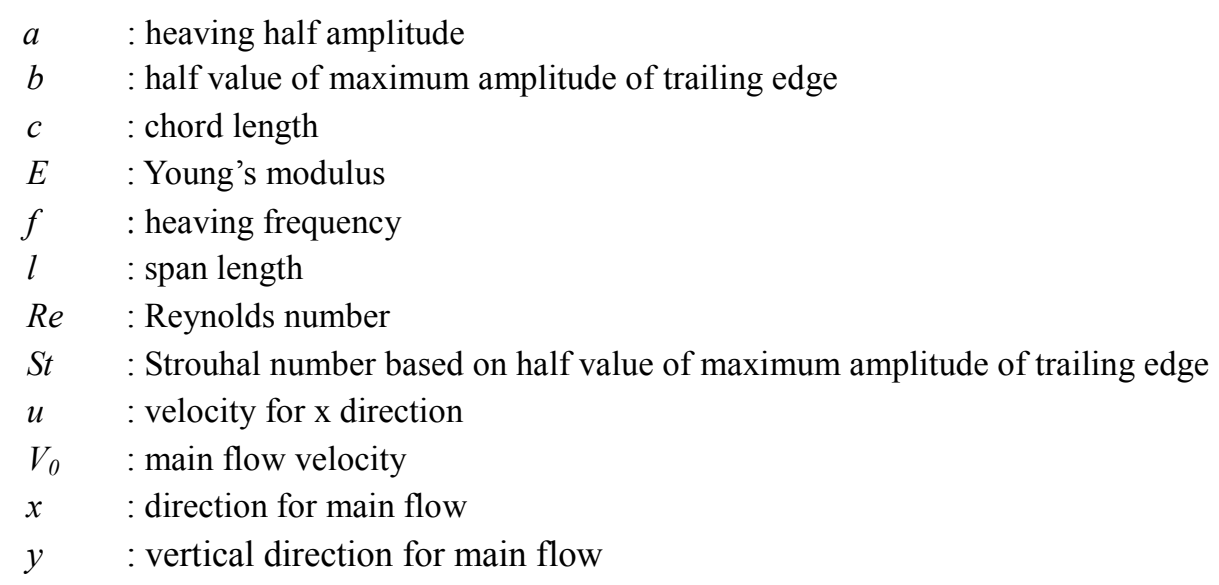

\section{Experimental setup}

\subsection{Elastic airfoil}

The test airfoils used in the present experiment were a rigid NACA0010 and an elastic NACA0010, as shown in Fig.1. The chord length and wing span length of both airfoils were $c=0.06 \mathrm{~m}$ and $l=0.20 \mathrm{~m}$, respectively, and the Reynolds number based on the chord length is 4,000 . The front and rear parts of the elastic NACA0010 were made from a stainless alloy $(E=205 \mathrm{GPa})$ and the silicon rubber $(E=0.32 \mathrm{MPa})$, respectively, and the ratio of the rigid 


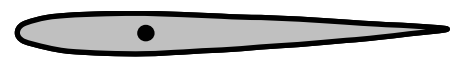

(a) Rigid NACA0010

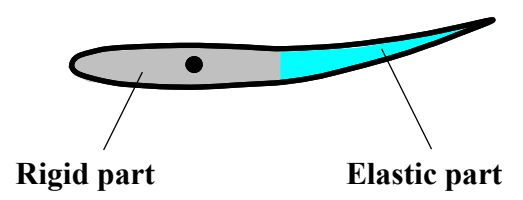

(b) Elastic NACA0010

Fig. 1 Test foil configuration used for experiment

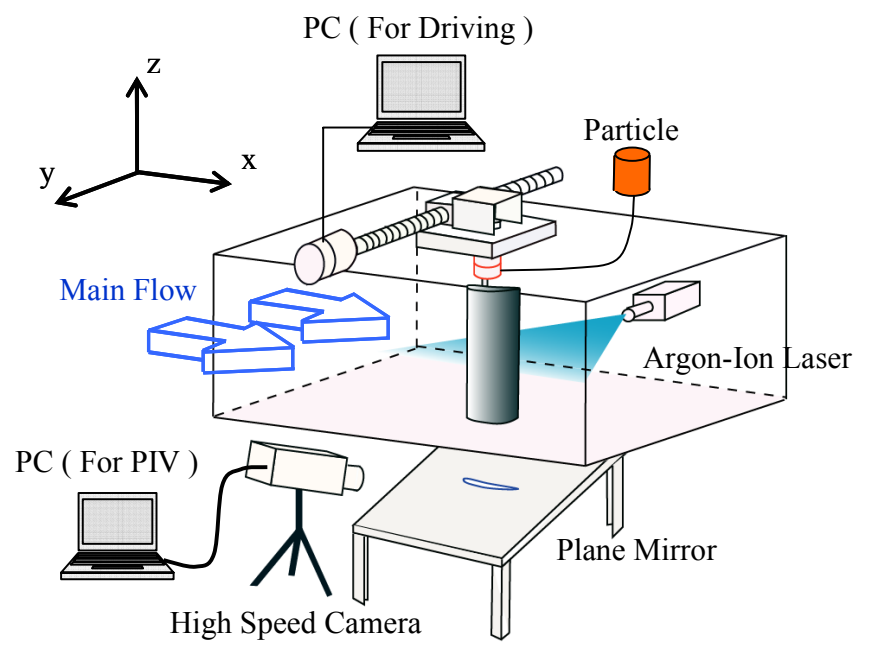

Fig. 2 PIV measurement systems

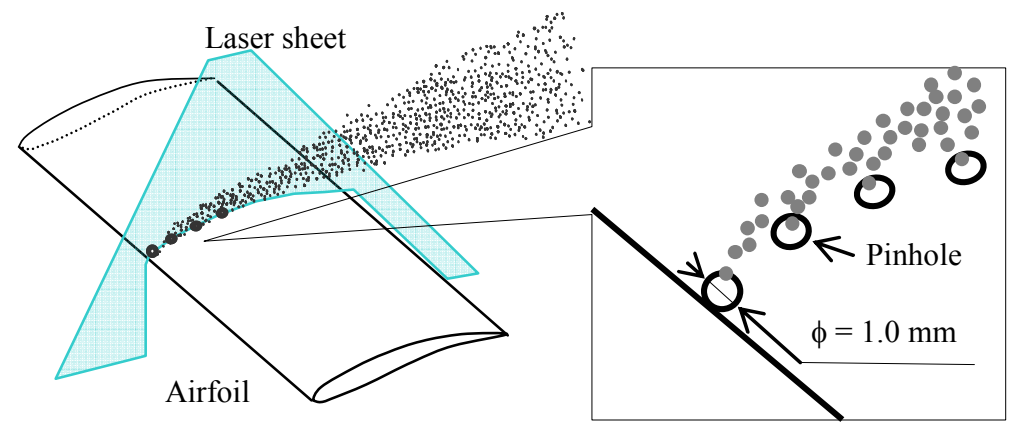

Fig. 3 Test foil with some pinhole

and elastic parts was 1:1. The length of both the rigid and elastic parts was $0.03 \mathrm{~m}$.

\subsection{PIV measurement system}

The PIV measurement equipment consisted of a water channel, a test airfoil, an excitation device, a water-cooled Ar-ion laser, a plane mirror and a high-speed camera, as shown in Fig. 2. The excitation device consisted of a DC servomotor and a ball screw. The clearance between the bottom of the water channel and the elastic airfoil was approximately $1.0 \mathrm{~mm}$, and the rolling up of three-dimensional vortices was controlled by placing a flat plate on the upper side of the water channel.

PIV measurements in the vicinity of the wall are important to reveal the growth process of vortices. However, in general, it is difficult to seed tracer particles sufficiently because a boundary layer develops on the surface of the wall. Therefore, we injected particles directly into the boundary layer from pinholes $(\phi=1.0 \mathrm{~mm})$ equipped at the front and on the 


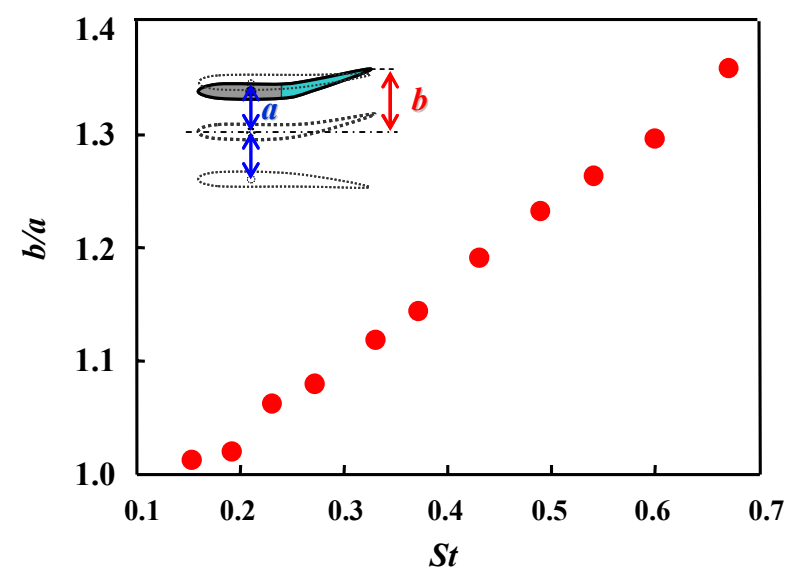

Fig. 4 Deformation value of trailing edge against Strouhal number

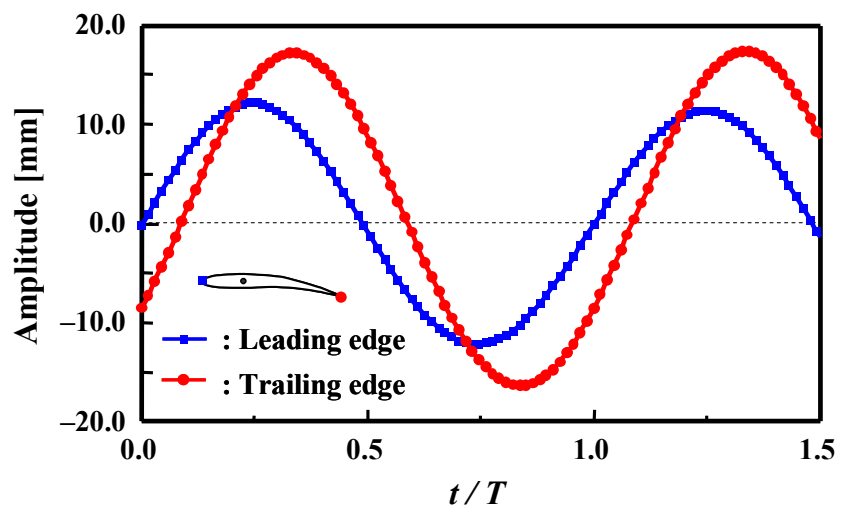

Fig. 5 Amplitude of leading and trailing edge of elastic NACA0010 at $S t=0.64$

surface on the airfoil, as shown in Fig. 3, and evaluated the vortex flow in the vicinity of the wall by PIV measurements. We used glass hollow spheres (Kanomax) having a diameter of $\phi=10 \mu \mathrm{m}$ as tracer particles. As shown in Fig. 3, we injected particles by setting the source of particles to appropriate location, such that particles are not to shed from pinholes. Heaving motions defined by Eq. (1) were applied to the test airfoil, and, as shown in Eq. (2), we defined the Strouhal number based on the half value of maximum amplitude of trailing edge. The heaving amplitude and the main flow are fixed at $12[\mathrm{~mm}]$ and $0.067[\mathrm{~m} / \mathrm{s}]$, respectively. We performed PIV measurements for $0.14<S t<0.70$ for heaving frequencies in the range of $0.4<f<1.5$.

$$
\begin{aligned}
& y=a \sin (2 \pi f t) \\
& S t=\frac{2 b f}{V_{0}}
\end{aligned}
$$

Figure 4 shows the deformation value of the trailing edge of the elastic NACA0010. The deformation value means the half value of maximum amplitude of trailing-edge nondimentionalized the heaving half amplitude. The abscissa indicates the Strouhal number based on the half value of maximum amplitude of the trailing edge, and the ordinate indicates the deformation value. The deformation value of the elastic NACA0010 increases as the Strouhal number increases, and the maximum deformation value is 1.36 times larger than heaving half amplitudes. We confirmed that elastic deformation was seen only at the first-order mode over the entire range of Strouhal number. 

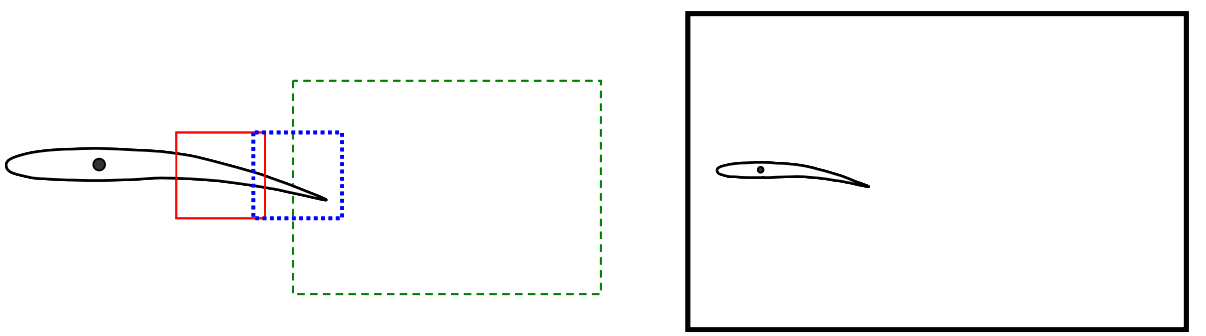

Fig. 6 Shooting domain for PIV measurement

Figure 5 shows the amplitude of the leading and trailing edges of the heaving elastic NACA0010 as a function of time at $S t=0.64$. The abscissa and ordinate indicate the nondimentionalized time $t / T$ and the amplitude of the heaving NACA0010, respectively. We confirmed that the amplitude of the trailing edge reached the maximum value just after the leading edge reached the top or bottom dead points. Therefore, the behaviors of the trailing edge are very different from those of the leading edge around the top dead point $(0.25<t / T$ $<0.35)$ or bottom dead point $(0.75<t / T<0.85)$ in heaving motions.

We captured images in four shooting domains for PIV measurements, as shown in Fig. 6 , in order to clarify the growth processes of vortices in the vicinity of the wall and wake structures. Red and blue areas indicate the shooting domains for the vortex flows developed in the vicinity of the wall, and green and black indicate the shooting domain for vortices rolled up from the trailing edge and wake structures, respectively. The time and special resolutions of the high-speed camera are $500 \mathrm{fps}$ and 1,024 $\times 1,024$ pixels, respectively, and we captured images for each shooting domain. Furthermore, when we shot the wake structure region defined by the black line in Fig. 6, we used ORGASAL (Kanomax) with a diameter of $50 \mu \mathrm{m}$ as tracer particles in order to obtain better PIV measurements from the viewpoint of the relationships between the shooting domain size and particle size in the images.

\section{Results and discussion}

\subsection{Vortex flow developed in the vicinity of the wall of heaving NACA0010}

The flow fields obtained from the vicinity of the wall for the heaving rigid NACA0010 and the elastic NACA0010 at $S t=0.64$ are shown in Figs. 7 and 8, respectively. The vorticity was calculated by the velocity which is subtracted the heaving velocity of airfoil from the velocity obtained by PIV measurement and Eq. (3). These results are instantaneous values at each location at the time that the NACA0010 heaved from the center point of the heaving amplitude $(y / c=0.0)$ to the bottom dead point $(y / c=0.2)$ and moved to the center point of the heaving amplitude $(y / c=0.0)$ again. In Figs. 7 and $8,(a),(b),(c)$ and $(d)$ show the results at $t / T=0.68,0.75,0.85$ and 0.92 , respectively.

$$
\omega=\frac{\partial v}{\partial x}-\frac{\partial u}{\partial y}
$$

As shown in Figs. 7 and 8, vortices with high vorticity are generated in the vicinity of the wall of the trailing edges of the heaving rigid NACA0010 and the elastic NACA0010. In addition, we have confirmed that these vortexes rolled up from the trailing edge after growing sufficiently on the wall. As shown in Fig. 7(a) and (b), the vorticity of the vortex generated in the vicinity of the rigid NACA0010 increases rapidly until the rigid NACA0010 arrives at the bottom dead point. Moreover, we also confirm that a vortex with high vorticity already rolls up from the trailing edge when the rigid NACA0010 arrived at the bottom dead point. In addition, as shown in Fig. 7(d), the vorticity of the vortex 


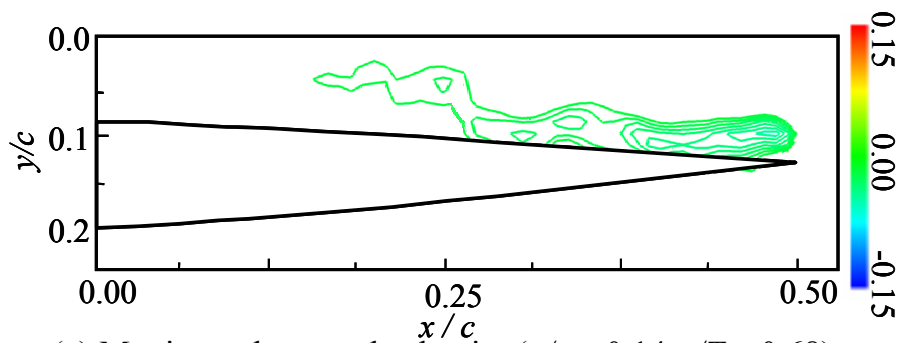

(a) Moving to bottom dead point $(y / c=0.14, t / T=0.68)$

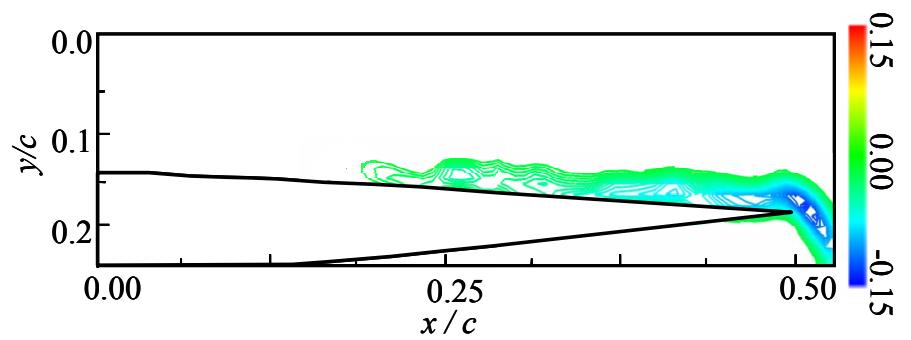

(b) Bottom dead point $(y / c=0.2, t / T=0.75)$

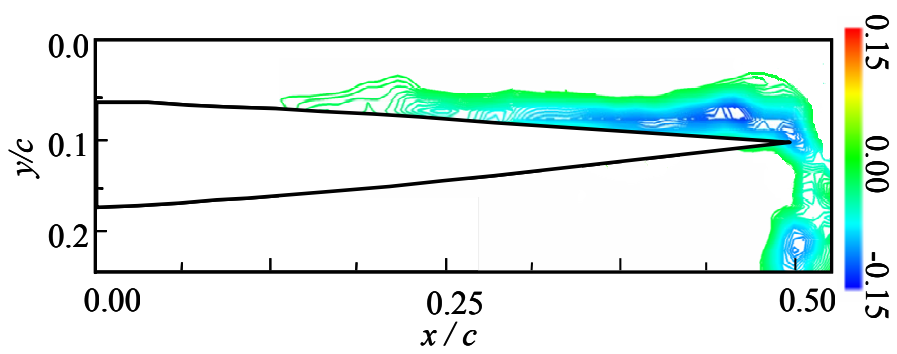

(c) Moving to top dead point $(y / c=0.12, t / T=0.85)$

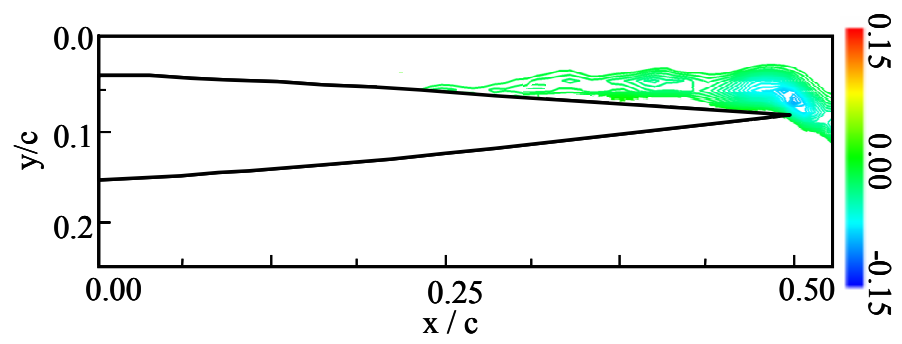

(d) Moving to top dead point $(y / c=0.08, t / T=0.92)$

Fig. 7 Instantaneous vorticity contour in the vicinity of the wall of heaving rigid NACA0010 at $S t=0.64$

generated in the vicinity of the wall tends to become weaker after the vortex rolled up from trailing edge.

Meanwhile, as shown in Fig. 8(a), the elastic NACA0010 always generates vortices over a larger area than the NACA0010 in the vicinity of the wall. In addition, we confirmed that the vortices generated in the vicinity of the wall of elastic NACA0010 do not increase rapidly at the bottom dead point (Fig. 8(b)). Furthermore, as shown in Fig. 8(c) and (d), we have confirmed the vortices that grow in the vicinity of elastic NACA0010 do not roll up at bottom dead point and that these vortices roll up during the heaving NACA0010 heaves from the bottom dead point to the center point of the heaving amplitude. Therefore, the increasing vorticity of the vortices generated in the vicinity of the wall and the phase at which the vortices roll up from the trailing edge are different in the rigid NACA0010 and the elastic NACA0010.

As shown in Fig. 5, the behaviors of the trailing edge are different from those of the 


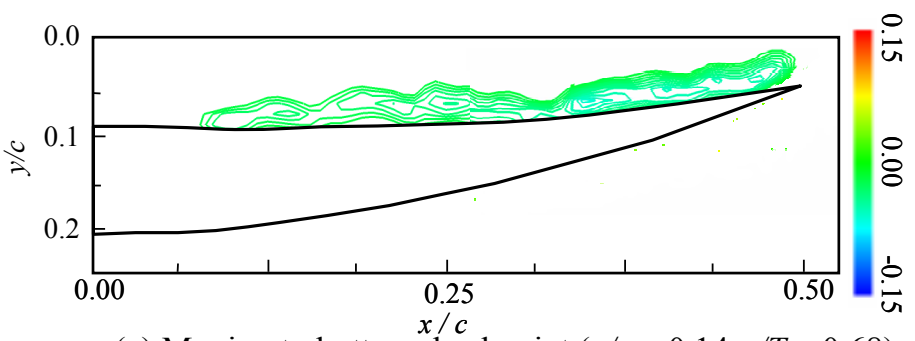

(a) Moving to bottom dead point $(y / c=0.14, t / T=0.68)$

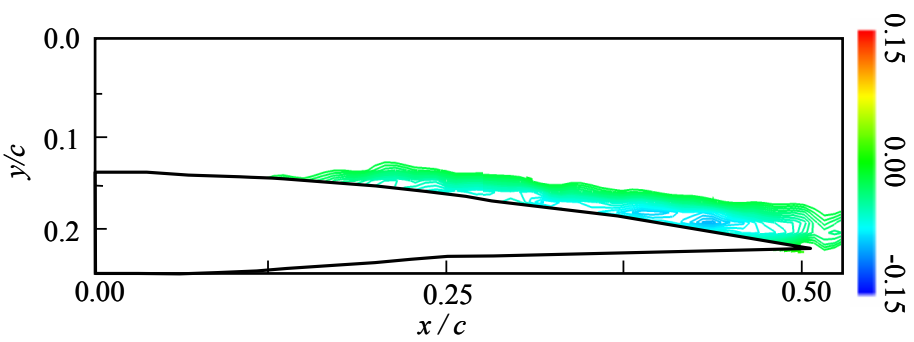

(b) Bottom dead point $(y / c=0.2, t / T=0.75)$

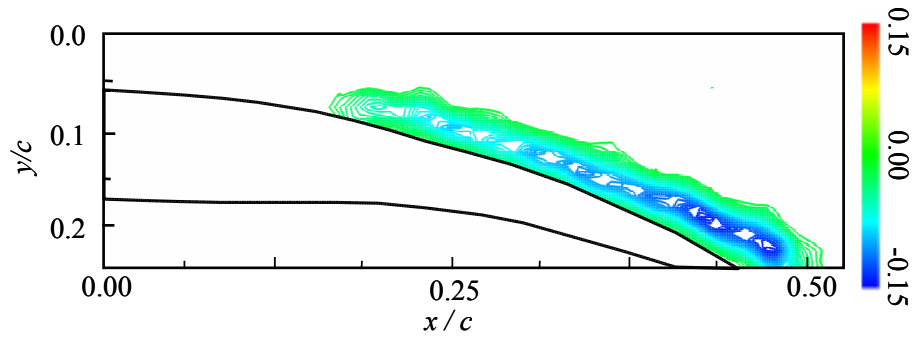

(c) Moving to top dead point $(y / c=0.12, t / T=0.85)$

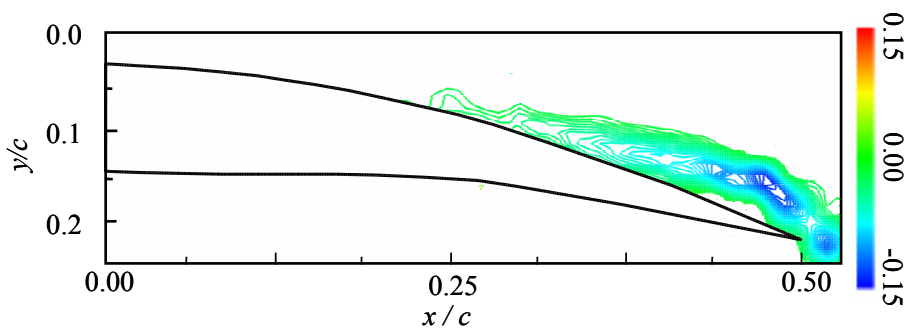

(d) Moving to top dead point $(y / c=0.08, t / T=0.92)$

Fig. 8 Instantaneous vorticity contour in the vicinity of the wall of heaving elastic NACA0010 at $S t=0.64$

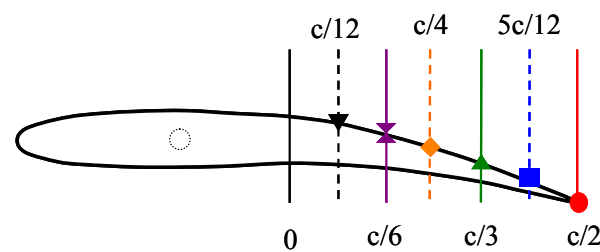

Fig. 9 Measurement point for instantaneous volume of vorticity in the vicinity of the wall of heaving NACA0010

leading edge. We believe that the differences in increasing process of vorticity of vortices generated in the vicinity of the wall and the phase at which the vortices roll up from the trailing edge occur because the behaviors of the trailing edge differ greatly from those of the leading edge in the range of $0.75<t / T<0.85$. 


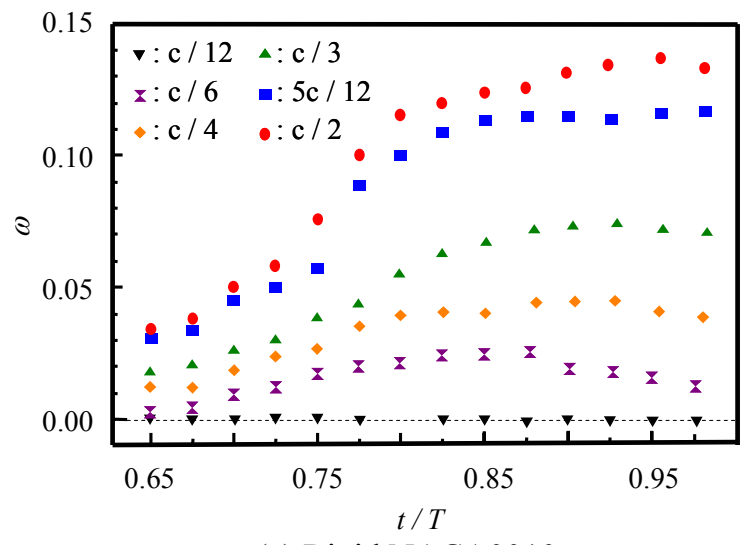

(a) Rigid NACA0010

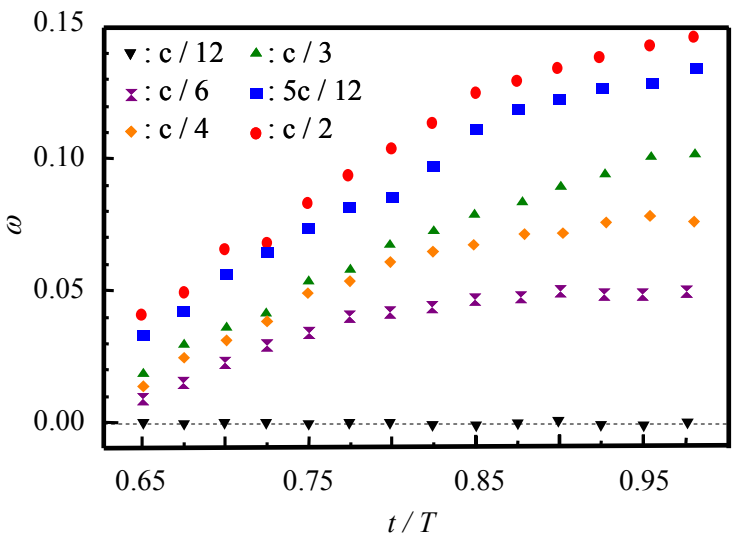

(b) Elastic NACA0010

Fig. 10 The vorticity change of vortex growing up in the vicinity of the wall

\subsection{Vorticity change of vortex generated in the vicinity of the wall for the heaving NACA0010}

In order to evaluate the growth processes of vortices that develop in the vicinity of the wall, we measured the vorticity at each of six measurement points $(x / c=c / 12, c / 6, c / 4, c / 3$, $5 c / 12, c / 2$ ) on the surface of the airfoil in the chord-wise direction, as shown in Fig. 9. The time history variations of the growth process of vortices in the vicinity of the wall for the heaving rigid NACA0010 and the elastic NACA0010 are shown in Figs. 10 (a) and (b), respectively. The abscissa and ordinate indicate the nondimentialized time $t / T$ and the vorticity, respectively.

As shown in Fig. 10 (a), the vorticity of vortices generated in the vicinity of the wall increases with time at all measurement points. Moreover, we have confirmed that the vorticity of vortices in the vicinity of the wall tends to increase toward the trailing edge. In particular, as the rigid NACA0010 heaves from the bottom dead point to the center point of the heaving amplitudes $(0.75<t / T<0.80)$, the vorticity of the vortices generated in the vicinity of the wall around the trailing edge $(x=5 \mathrm{c} / 12, \mathrm{c} / 2)$ increases rapidly. In addition, we confirmed that the vorticity tends to become weaker after the rapid growth.

On the other hand, as shown in Fig. 10 (b), the vorticity of the vortices generated in the vicinity of the wall increases with time, which is the same as the rigid NACA0010. However, this vorticity is different from that of the rigid NACA0010, the vorticity of the vortices generated in the vicinity of the wall for the elastic NACA0010 constantly increase with time, not only from the trailing edge, but also from around the center point of the chord of elastic NACA0010 to the trailing edge $(\mathrm{c} / 4<x<\mathrm{c} / 2)$. Moreover, the vorticity of vortex 


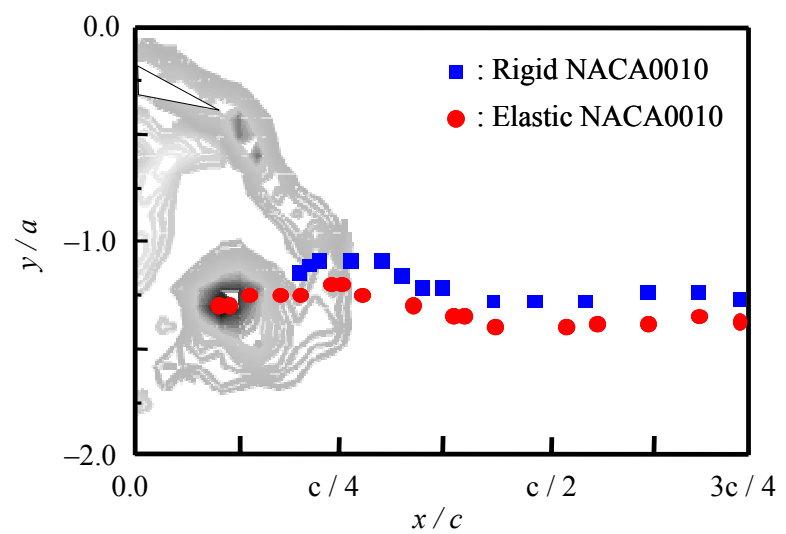

Fig.11 The trajectory of vortex rolled up in the wake of heaving NACA0010 at $S t=0.64$

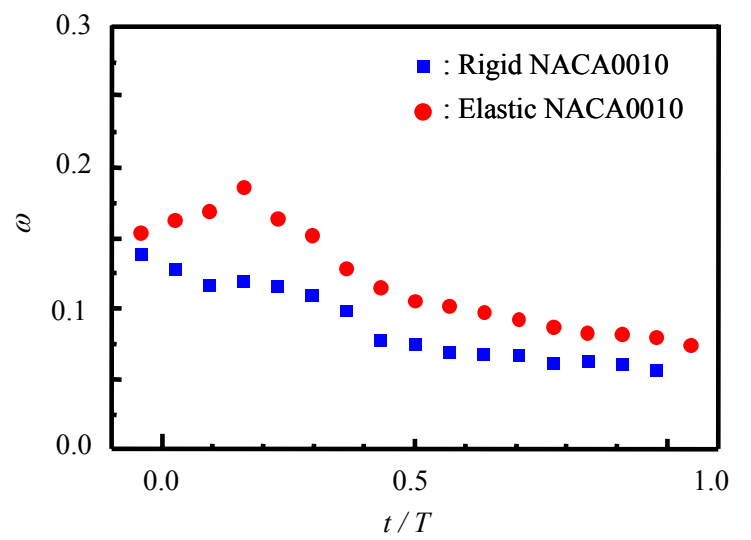

Fig.12 The vorticity change of vortex rolled up from trailing edge in the wake of heaving NACA0010

generated in the vicinity of the wall for the elastic NACA0010 does not increase rapidly at the bottom dead point and tends to increase more in the range of $t / T>0.80$. The elastic NACA0010 generates vortices at the elastic deformation part of the airfoil by elastic deformation and increases the vorticity of the vortices generated in the vicinity of the wall. Therefore, the elastic NACA0010 rapidly suppresses the increase in vorticity at the bottom dead point and encourages the further increase in vorticity with time. Therefore, the elastic NACA0010 generates vortices at the deformation part and increases this vorticity by elastic deformation. In addition, the elastic NACA0010 prevents the rapid increase at the bottom dead point and helps to increase the vorticity with time by elastic deformation.

\subsection{Behavior of vortex rolled up from trailing edge of heaving NACA0010}

In order to evaluate the behaviors of vortices rolled up from the trailing edge and grown in the wake, we measured the center point of the vortices and the time histories variation of vortices. The trajectories of vortex centers that rolled up from the trailing edge of the rigid NACA0010 and the elastic NACA0010 are shown in Fig. 11. The abscissa and ordinate indicate the nondimentiorized distance of the main flow direction $x / c$ and amplitude $y / a$, respectively. In this figure, $\square$ and indicate the center point of the vortices rolled up in the wake of the rigid NACA0010 and the elastic NACA0010, respectively.

The vortices rolled up from each NACA0010 are linearly swept away in the $x$-direction. Furthermore, the vortices that rolled up from the trailing edge of the elastic NACA0010 are separated at the location $(x / c=0.10)$, which is near the trailing edge for the $x$-direction and far from the center point of heaving amplitude for the $y$-direction, as compared with results for the rigid NACA0010 $(x / c=0.20)$. Therefore, the elastic NACA0010 forms a wake 


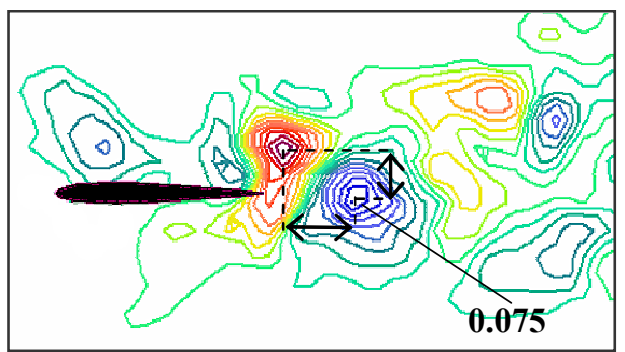

(a) Rigid NACA0010

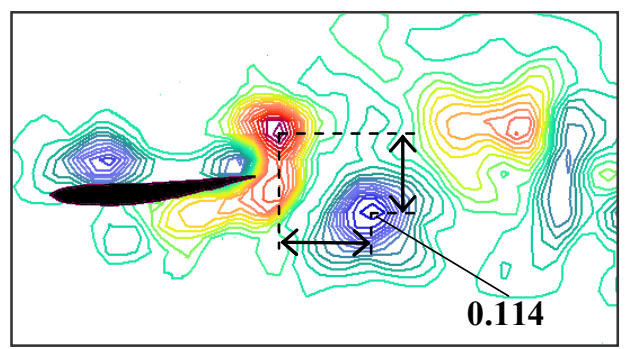

(b) Elastic NACA0010

Fig. 13 Instantaneous vorticity contour obtained from the wake of heaving NACA0010 at $t / T=0.50$ with $S t=0.64$

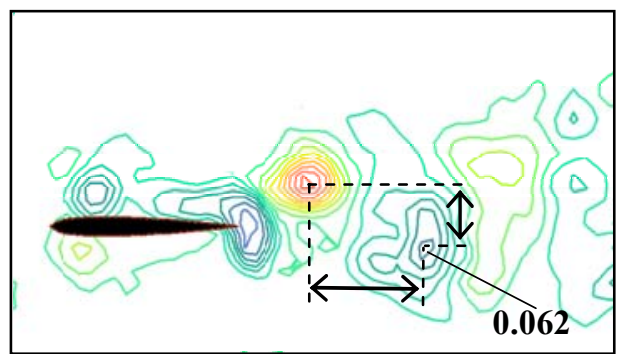

(a) Rigid NACA0010

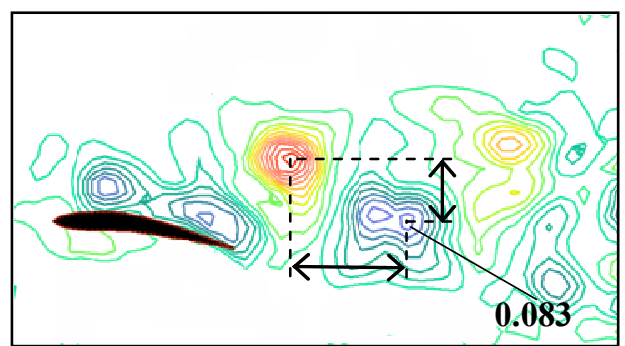

(b) Elastic NACA0010

Fig. 14 Instantaneous vorticity contour obtained from the wake of heaving NACA0010 at $t / T=0.85$ with $S t=0.64$

structure with wide vortex intervals in the wake.

The variations of the time histories of vorticity of the vortices that rolled up from the trailing edge of the rigid NACA0010 and the elastic NACA0010 are shown in Fig. 12. The abscissa and ordinate indicate the nondimentionalized time $t / T$ and the vorticity, respectively. In this figure, $\square$ and indicate the vorticity of the vortices that rolled up in the wake of the rigid NACA0010 and the elastic NACA0010, respectively.

The vorticity of the vortices that rolled up from the trailing edge of the rigid NACA0010 decreases with time. However, we have confirmed that vorticity of the vortices the trailing edge by means of elastic deformation, as shown in Fig. 10. In addition, the vorticies that rolled up from the elastic NACA0010 continue to grow for a time in the wake, 


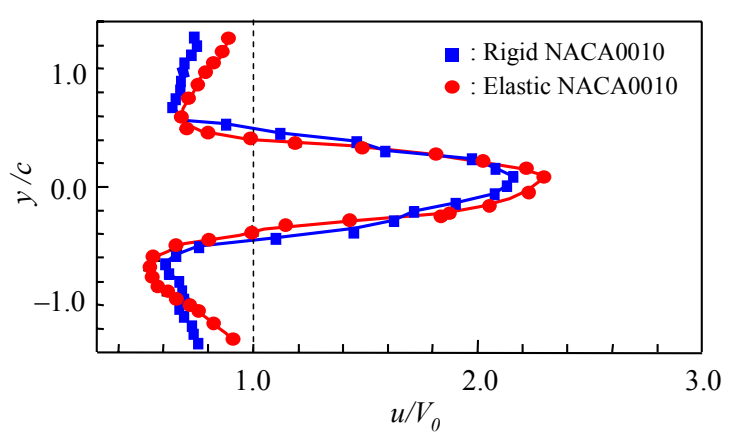

Fig. 15 Mean velocity profile one-chord length behind the heaving airfoils for one cycle at $S t=0.64$

even after the vortices roll up from the trailing edge. We assume that this is because the vortices generated in the vicinity of the wall continue to increase gradually until they roll up from difference in vorticity between the rigid NACA0010 and the elastic NACA0010 reaches a maximum value of $58 \%$ at $t / T=0.12$. However, we confirmed that the vorticities in the wake of the rigid NACA0010 and the elastic NACA0010 tend to exhibit the same behavior over time.

\subsection{Vortex flow patterns behind heaving airfoils}

Vorticity contours obtained from the wakes of the heaving rigid NACA0010 and the elastic NACA0010 at $t / T=0.50$ with $S t=0.64$ are shown in Figs. 13(a) and (b), respectively. These results are the instantaneous values at $y=0.0$ when NACA0010 heaves from the top dead point to the bottom dead point. The value and the arrow indicate instantaneous vorticity and the interval of the vortex that formed the wake structure, respectively.

Thrust producing vortex streets for which the array is opposite from that of a Karman vortex street is clearly formed in the wake of the rigid NACA0010 and the elastic NACA0010. Moreover, the instantaneous vorticity of the elastic NACA0010 is higher than that of the vortices forming the thrust producing vortex streets in the wake of the rigid NACA0010. In addition, the vortex streets are not formed as closely as those of the rigid NACA0010. Since the vortices formed thrust producing vortex streets, the vortices develop self sufficiency in the vicinity of the wall and are separated from the trailing edge far from the center point of the heaving amplitude.

Vorticity contours obtained from the wakes of the heaving rigid NACA0010 and the elastic NACA0010 at $t / T=0.85$ with $S t=0.64$ are shown in Figs. 14(a) and 14(b), respectively. The value and the arrow indicate instantaneous vorticity and the interval of vortex which formed wake structure, respectively.

Unlike the result shown in Fig. 13, we confirmed that the wake structures of the rigid NACA0010 and the elastic NACA0010 become similar. This is because the vorticity and the location of vortex become similar, as shown in Figs. 11 and 12.

\subsection{Accelerating flow one-chord length behind heaving airfoils}

Figure 15 shows the mean velocity profile in the $x$ direction one-chord length behind the heaving airfoil for one cycle. In this figure, $\mathbf{a}$ and indicate the results of the rigid NACA0010 and elastic NACA0010, respectively. The abscissa and ordinate indicate the nondimensionalized flow velocity $u / V_{0}$ and the amplitude $y / c$, respectively. Here, $u / V_{0}>1.0$ indicates an accelerating flow.

We confirmed that the accelerating flow profiles in the wake of the rigid NACA0010 and the elastic NACA0010 are approximately the same. As shown in Fig. 13, the 
instantaneous values of the vorticity of the vortex and the flow pattern are different in the rigid NACA0010 and the elastic NACA0010. Therefore, the instantaneous accelerating flow induced by the vortex streets and the region of the velocity deficit are different in the rigid NACA0010 and the elastic NACA0010. This is because the growth process of vorticity on the wall is different until the vortices roll up from the trailing edge, as shown in Figs. 7, 8, and 10. However, as shown in Figs. 12 and 13, the flow fields in the wake of the rigid NACA0010 and the elastic NACA0010 tend to have a similar structure over time. This is because the vorticity of the elastic NACA0010 was swept into the wake and decreased over time. Therefore, the vortex flow in the vicinity of the wall and the process of wake structure formation differ between the rigid NACA0010 and the elastic NACA0010. However, the wake structures for which the flow fields are averaged for one heaving cycle between the rigid NACA0010 and the elastic NACA0010.

\section{Concluding remarks}

The instantaneous vorticity of the vortices forming the thrust producing vortex streets in the wake of the elastic NACA0010 is higher than that of the vortices forming the thrust producing vortex streets in the wake of the rigid NACA0010, and the vortex streets for the elastic NACA0010 are not formed as closely as those for the rigid NACA0010. Since the vortices formed thrust producing vortex streets, the vortices develop self sufficiency in the vicinity of the wall of the elastic NACA0010 and are separated from the trailing edge far from the center point of heaving amplitude. However, the flow fields in the wake of the rigid NACA0010 and the elastic NACA0010 tend to have similar structures over time. Therefore, the vortex flow in the vicinity of the wall and the wake structure formation process differ between the rigid NACA0010 and the elastic NACA0010, but the wake structures for which the flow fields are averaged for one heaving cycle become approximately the same in the wake of the rigid NACA0010 and the elastic NACA0010.

\section{Acknowledgements}

This work was supported by Grant-in-Aid for Scientific Research (B) 22360080.

\section{References}

(1) Katzmayr, R., Effect of periodic changes of angle of attack on behaviour of airfoils, NACA 147., 1922

(2) Jones,K.D.etal. Jones, K.D., Dohring, C.M., Platzer, M.F., Experimental and computational investigation of the Knoller-Betz effect, AIAA Journal, Vol. 36, No.7 (1998), pp.1240-1246

(3) Lai,J.C.S. et al. Platzer, M.F., Jet characteristics of a plunging airfoil, AIAA Journal, Vol.37, No.12 (1999), pp.1529-1537

(4) Ramamurti, R. Sandberg, W.C., Simulation of flow about flapping airfoils using finite element incompressible flow solver, AIAA Journal,, Vol.39, No.2 (2001), pp.253-260.

(5) Fuchiwaki, M.Tanaka K., Two Dimentional Structure of the Wake behind a Pitching Airfoil with Higher Non-dimentional Pitching Rate, 10 th International Symposium on Flow Visuakization, ( 2002 ) F0107

(6) Fuchiwaki, M. Tanaka K., Nakashima M., Characteristics of Dynamic Thrust on an Unsteady Airfoil in Pitching and Heaving Motions, ASME Fluid Engneering Division Summer Meeting, (2007) ASME FEDSM 2007-37222

(7) Taylor G.K. Nudds, R.L., Thomas, A.L.R., Flying and swimming animals cruise at a Strouhal number tuned for high power efficiency, Nature 425 (2003), pp.707-711.

(8) Heathcote, S., Martin D., Gursul I., Flexible Flapping Airfoil Propulsion at Zero Freestream Velocity, AIAA Journal, Vol. 42, No.11 (2005), pp.2197-2204.

(9) Heathcote S., Gursul I., Flexible Flapping Airfoil Propulsion at Low Reynolds Numbers, AIAA Journal, Vol. 45, No.5 (2007), pp.1067-1079. 
(10) Young Y. L., Fluid-structure interaction analysis of flexible composite marine propellers Journal of Fluid and Structure, Vol. 24 (2008), pp. 799-818 